\title{
An Improved Physics-Based Formulation of the Microwave p-i-n Diode Impedance
}

\author{
Emmanuel Gatard, Raphaël Sommet, Philippe Bouysse, and Raymond Quéré, Senior Member, IEEE
}

\begin{abstract}
An improved formulation of the frequency-dependent impedance for p-i-n diodes from physical and geometrical parameters is presented. This work is addressed to diode designers and allows them to evaluate quickly and accurately the diode impedance. It comes in parallel with existing SPICE p-i-n diode model [1] used in CAD software. Under forward bias conditions, important recombinations occur in the heavily doped end regions of thin $\mathrm{p}-\mathrm{i}-\mathrm{n}$ diodes that seriously affects the diode impedance. This effect is taken into account to increase the accuracy of existing numerical models and to extend their validity domain to any I-region thicknesses. This improvement has been validated by measurement results on a 5- $\mu \mathrm{m}$ I-region width silicon p-i-n diode.
\end{abstract}

Index Terms-Bias-dependent impedance, carrier lifetime, forward bias, p-i-n diode, recombinations.

\section{NOMENCLATURE}

$A$

$I(t), i_{0}, \tilde{i}$

$P(x, t)$

$p_{0}, \tilde{p}$

$e_{0}, \tilde{e}$

$W$

$\mu_{n}, \mu_{p}$

$b=\mu_{n} / \mu_{p}$

$\tau_{I}$

$\tau_{P+}, \tau_{N+}$

$h_{p}, h_{n}$

$D_{a}$

$L_{a}=\sqrt{D_{a} \tau_{I}}$

$L_{a_{\mathrm{eff}}}=L_{a} / 1+j \omega \tau_{I}$

$V_{T}=k T / q$
Active diode section.

Total, dc and ac diode currents.

Total carrier density in the base.

DC and ac base carrier densities.

DC and ac electric fields in the base.

I-region thickness.

Electron and hole mobilities.

Electron to hole mobility ratio.

Base ambipolar carrier lifetime.

Emitter carrier lifetimes.

Emitter recombination parameters.

Ambipolar diffusion constant.

DC ambipolar diffusion length.

AC effective diffusion length.

Thermal voltage.

\section{INTRODUCTION}

$\mathbf{P}$ -I-N diodes are widely used in active, passive microwave applications including phase shifters, switches, attenuators and limiters. Applications at microwave frequencies, as well as lower frequencies, require a good estimation of the diode impedance to correctly determine the insertion loss or phase shift. Under forward bias conditions, the impedance is governed by the stored charge in the I-region (base) due to dc and ac drive currents [2]. At low frequencies, carrier density variations

Manuscript received September 8, 2006; revised October 30, 2006. This work was supported by Chelton Telecom and Microwave, Les Ulis, France and Thales Air Defence, Rouen Ymare, France.

The authors are with the XLIM CNRS Research Laboratory, Department of High Frequency Components Circuits Signals and Systems, Brive la Gaillarde 19100, France (e-mail: emmanuel.gatard@xlim.fr).

Digital Object Identifier 10.1109/LMWC.2006.890483 take place in the entire I-region. In this case, the overall diode impedance is dominated by resistive and reactive effects introduced by the two junctions. The I-region acts like an electrical short circuit at very low frequencies [3]. At higher frequencies, the ac injected charge is concentrated only near the junctions. For this reason, junctions act at these frequencies like short circuits, and thus, the total diode impedance is dominated by the conductivity modulation of the base.

The diode impedance, according to physics based equations, depends on the diode geometry and semiconductor properties [4]. An accurate small signal numerical model based on geometrical and physical parameters (electron and hole mobilities, carrier lifetime and I-region thickness) has been developed to determine the diode impedance-frequency characteristics of the forward bias p-i-n diode [3]. The Caverly standard model [3] only supposes a recombination current in the I-region. This assumption is valid for most cases, especially for diodes with thick I-region. In the case of thick diodes, most injected carriers recombine in the base and do not reach the end regions. However, for $\mathrm{p}$-i-n diodes with small base length to diffusion length $\left(W / L_{a}\right)$ ratio, recombinations in the heavily doped end regions [5], [6] seriously affect the total diode impedance. In this case, the diode impedance is overestimated at low frequencies and underestimated at high frequencies.

This letter provides physics-based expressions and proves that it is possible to accurately compute the small signal diode impedance for any I-region thicknesses from technological and material parameters. Thus, this model can be used to optimize not only the overall diode impedance but also the base impedance or each junction impedance separately with a reduced computation time.

\section{MODEL DESCRIPTION}

In the case of thin p-i-n diodes, recombinations in the heavily doped $\mathrm{p}^{+}$and $\mathrm{n}^{+}$end regions are negligible at low current densities. At high current densities, they become significant and reduce the two junction impedances. This phenomenon is illustrated on Fig. 1.

In order to extend the validity domain of the standard numerical model [3] to any kind of p-i-n diodes, it becomes necessary to take into account all of these recombination currents. The base impedance and the two junction impedances are calculated from the carrier distribution in the I-region. Assuming equal electron and hole concentrations and Shockley-Read-Hall recombinations, the carrier distribution in the intrinsic region is governed by the ambipolar diffusion equation:

$$
D_{a} \frac{\partial^{2} P(x, t)}{\partial x^{2}}=\frac{P(x, t)}{\tau_{I}}+\frac{\partial P(x, t)}{\partial t} .
$$




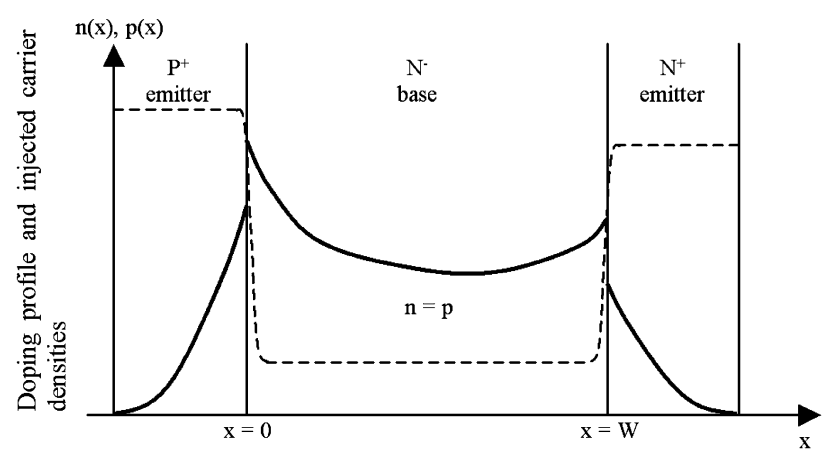

Injected holes

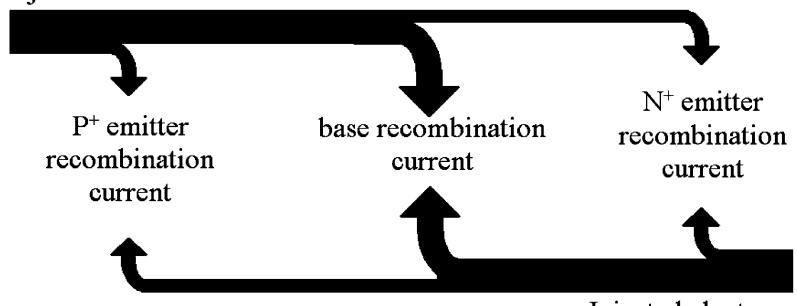

Injected electrons

Fig. 1. Schematic representation of doping profile (dashed line) and injected carrier densities (continuous curve) and recombination process in the $\mathrm{p}-\mathrm{i}-\mathrm{n}$ diode.

To take into account recombination currents in the emitters, the boundary conditions for $(1)$ at the $\mathrm{P}^{+}-\mathrm{I}(x=0)$ and $\mathrm{I}-\mathrm{N}^{+}(x=$ $W)$ junctions can be written as [7]

$$
\begin{aligned}
\frac{\partial P}{\partial x}(0, t) & =-\frac{b}{1+b} \frac{I(t)}{q A D_{a}}+\frac{h_{p}}{D_{a}} P(0, t)^{2} \\
\frac{\partial P}{\partial x}(W, t) & =\frac{1}{1+b} \frac{I(t)}{q A D_{a}}-\frac{h_{n}}{D_{a}} P(W, t)^{2}
\end{aligned}
$$

where $h_{p}$ and $h_{n}$ are emitter recombination parameters defined in [7]. It has been demonstrated that recombination currents in the end regions can be described by the $\left(h_{p, n} / D_{a}\right) P(x, t)^{2}$ term [5] for each boundary condition in (2) and (3). These additional terms improve the accuracy of the total diode impedance, especially in the case of thin $\mathrm{p}-\mathrm{i}-\mathrm{n}$ diodes. In the previous work described in [3], these terms were not taken into account. The carrier density $P(x, t)$ is defined as the sum of dc and ac charge density components

$$
P(x, t)=p_{0}(x)+\tilde{p}(x) \exp (j \omega t) .
$$

Assuming a small signal approach for all quantities, dc and ac carrier distributions in the base are, respectively, given by

$$
\begin{aligned}
p_{0}(x)= & \frac{L_{a}}{\sinh \left(\frac{W}{L_{a}}\right)}\left[\left(\frac{1}{1+b} \frac{i_{0}}{q A D_{a}}-\frac{h_{n}}{D_{a}} p_{0}(W)^{2}\right)\right. \\
& \times \cosh \left(\frac{x}{L_{a}}\right)-\left(-\frac{b}{1+b} \frac{i_{0}}{q A D_{a}}+\frac{h_{p}}{D_{a}} p_{0}(0)^{2}\right) \\
& \left.\times \cosh \left(\frac{W-x}{L_{a}}\right)\right]
\end{aligned}
$$

$$
\begin{aligned}
\tilde{p}(x)= & \frac{L_{a_{\mathrm{eff}}}}{\sinh \left(\frac{W}{L_{a_{\mathrm{eff}}}}\right)}\left[\left(\frac{1}{1+b} \frac{\tilde{i}}{q A D_{a}}-\frac{h_{n}}{D_{a}} 2 p_{0}(W) \tilde{p}(W)\right)\right. \\
& \times \cosh \left(\frac{x}{L_{a_{\mathrm{eff}}}}\right) \\
& -\left(-\frac{b}{1+b} \frac{\tilde{i}}{q A D_{a}}+\frac{h_{p}}{D_{a}} 2 p_{0}(0) \tilde{p}(0)\right) \\
& \left.\times \cosh \left(\frac{W-x}{L_{a_{\mathrm{eff}}}}\right)\right] .
\end{aligned}
$$

The dc carrier concentrations $p_{0}(0)$ and $p_{0}(W)$ near the two junctions are required to calculate the junction and the base impedances. They are obtained by solving the nonlinear system written from (5) at $(x=0)$ and $(x=W)$. The ac components $\tilde{p}(0)$ and $\tilde{p}(W)$ are computed at each frequency by solving the linear system given by (6) at the two junctions. The frequency-dependent impedance $Z_{T}$ of the diode is then defined as the sum of the two junction impedances $Z_{j_{P-I}}, Z_{j_{I-N}}$ and the base impedance $Z_{I}$ when ohmic contacts are neglected

$$
Z_{T}=Z_{j_{P-I}}+Z_{j_{I-N}}+Z_{I}
$$

From junction impedance expressions established in [4], $P-I$ and $I-N$ junction impedances are given by

$$
\begin{aligned}
Z_{j_{P-I}} & =\frac{V_{T}}{\tilde{i}} \frac{\tilde{p}(0)}{p_{0}(0)} \\
Z_{j_{I-N}} & =\frac{V_{T}}{\tilde{i}} \frac{\tilde{p}(W)}{p_{0}(W)} .
\end{aligned}
$$

The base impedance expression is defined from conduction and diffusion currents of the electrons and holes into the base. The total current in the base can be written as

$$
\begin{aligned}
I(t)= & i_{0}+\tilde{i} \\
= & q A\left(p_{0}(x)+\tilde{p}(x)\right)\left(\mu_{n}+\mu_{p}\right)\left(e_{0}(x)+\tilde{e}(x)\right) \\
& +q A V_{T}\left(\mu_{n}-\mu_{p}\right) \frac{\partial\left(p_{0}(x)+\tilde{p}(x)\right)}{\partial x} .
\end{aligned}
$$

The ac component $\tilde{e}(x)$ of the electric field is extracted from (10). Its integration along the base leads to the ac voltage drop $\tilde{v}[8]$. Finally, the impedance of the base region can be given by

$$
\begin{aligned}
Z_{I}= & \frac{\tilde{v}}{\tilde{i}}=\int_{0}^{W} \frac{1}{q A\left(\mu_{n}+\mu_{p}\right) p_{0}(x)} d x \\
& -\int_{0}^{W} \frac{i_{0}}{\tilde{i}} \frac{1}{q A\left(\mu_{n}+\mu_{p}\right)} \frac{\tilde{p}(x)}{p_{0}(x)^{2}} d x \\
& +\frac{V_{T}}{\tilde{i}} \frac{\mu_{n}-\mu_{p}}{\mu_{n}+\mu_{p}}\left[\frac{\tilde{p}(0)}{p_{0}(0)}-\frac{\tilde{p}(W)}{p_{0}(W)}\right] .
\end{aligned}
$$

Recombination in the emitters of the p-i-n diode modifies the boundary conditions of the ambipolar diffusion equation (1). This implies a change of the base carrier density given now by (5) and (6). As for the junction and the base impedance expressions, they stay unchanged whether you consider recombinations or not in the emitters. The model described here is suitable for any base region thicknesses. 

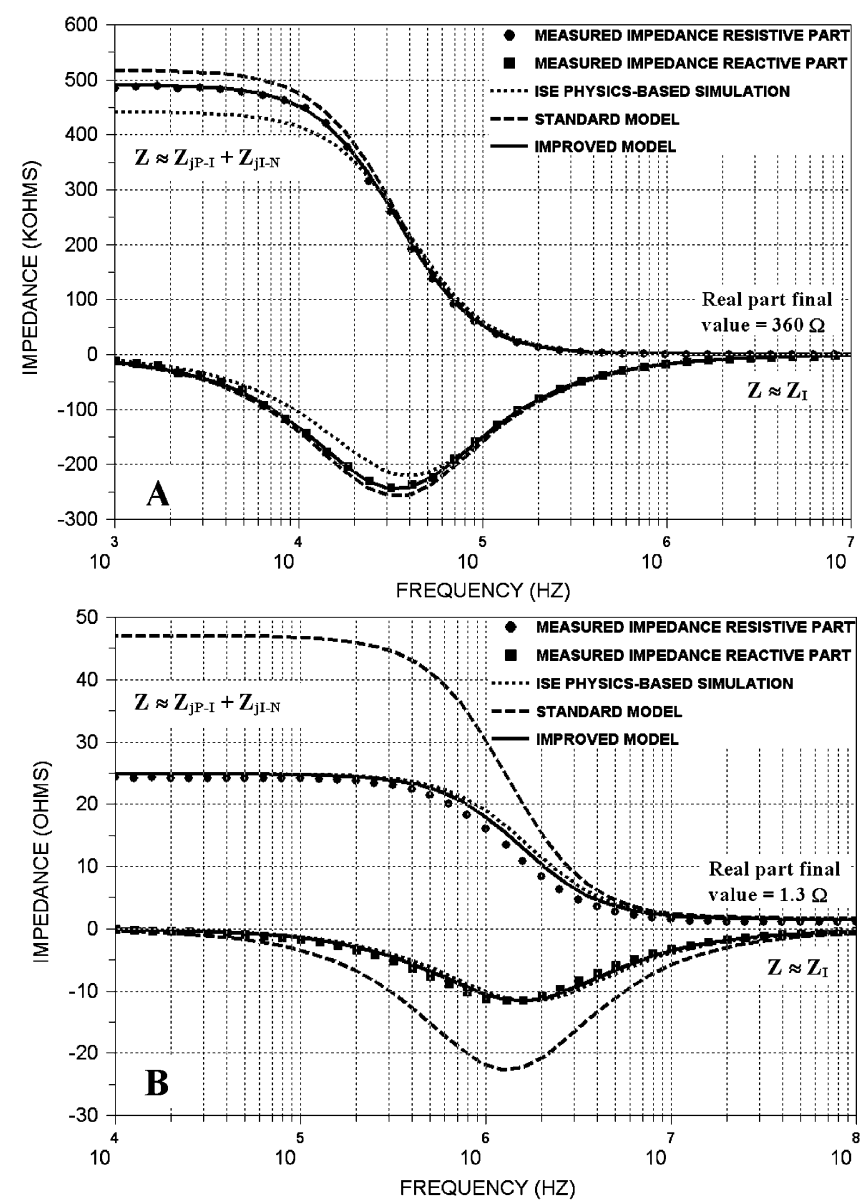

Fig. 2. Comparison between measured and calculated resistance/reactance in the case of physics-based simulations, the standard and improved models. In (a), measurement has been made for a low dc bias current of $0.1 \mu \mathrm{A}$ which corresponds to $\tau_{\text {eff }}=5.2 \mu \mathrm{s}$. In the case of (b), the dc bias current is now high and equal to $1.1 \mathrm{~mA}$ which corresponds to $\tau_{\text {eff }}=120 \mathrm{~ns}$. The improved model results were obtained with the following parameters: $h_{n}=1.6 \times 10^{-22} \mathrm{~m}^{4} / \mathrm{s}$, $h_{p}=1.9 \times 10^{-22} \mathrm{~m}^{4} / \mathrm{s}, W=5 \mu \mathrm{m}, \tau_{I}=5.2 \mu \mathrm{s}, A=4 \times 10^{-9} \mathrm{~m}^{2}$.

\section{EXPERIMENTAL RESULTS}

The proposed model has been validated on a thin silicon $\mathrm{p}-\mathrm{i}-\mathrm{n}$ diode of 5- $\mu \mathrm{m}$ I-region width, from Chelton Telecom and Microwave foundry. The reactive part of the diode impedance exhibits a minimum which occurs for a frequency $f=1 /\left(2 \pi \tau_{\text {eff }}\right)$ [9]. $\tau_{\text {eff }}$ is called the effective carrier lifetime and depends on bias level. Whereas the carrier lifetimes $\tau_{P+}, \tau_{I}, \tau_{N+}$ are independent of bias current, the effective carrier lifetime $\tau_{\text {eff }}$ seen at the device terminals is nonlinear due to the nonlinearity of the stored charge variation with the bias currents $\left(Q=i_{0} \tau_{\text {eff }}\right)$ [6]. The resistive and reactive parts of the total diode impedance have been measured for two dc bias currents and compared with our improved model, the standard numerical model [3], and physics-based simulations in Fig. 2. In the case of 1-D physics-based simulations with the ISE TCAD software, the contact effects are also neglected. However, the results in Fig. 2 show that the accuracy is not affected by this assumption. At a low bias current of $0.1 \mu \mathrm{A}$ [Fig. 2(a)], recombinations in the end regions are negligible thus the measured carrier lifetime of $5.2 \mu$ s corresponds to the I-region carrier lifetime $\tau_{\text {eff }}=\tau_{I}$. In this case, the two numerical models exhibit a good agreement with measurement and ISE results. With an higher bias current of $1.1 \mathrm{~mA}$ [Fig. 2(b)], the minimum diode reactance takes place now for a frequency greater than $1 \mathrm{MHz}$. $\tau_{\text {eff }}$ decreases now with the bias current due to recombination in the end regions and becomes a complex function which depends on diode physical and technological parameters $\left(h_{n}, h_{p}, W, \tau_{I}\right.$ and $\left.i_{0}\right)$ [6]. When emitter recombination takes place, the standard model is unable to predict with accuracy the diode impedance. Moreover, the knowledge of the effective carrier lifetime for each desired bias current is required. With the improved model, the diode impedance and the current-dependent carrier lifetime law are predicted with accuracy without having to set the carrier lifetime for each bias current.

\section{CONCLUSION}

An improved bias-dependent small signal model has been presented in order to predict with accuracy the impedance-frequency behavior for thin p-i-n diodes under forward bias conditions. This improvement has been validated with measurements and physics-based simulations. The proposed model is suitable for any I-region thicknesses and the current-dependent carrier lifetime is automatically taken into account thanks to recombination currents in the end regions. This work allows diode designers to compute accurately and quickly the diode impedance from technological and material parameters. The Scilab implementation of this work is available on request to the authors. A SPICE model has also been developed to allow circuit designers to predict $\mathrm{p}-\mathrm{i}-\mathrm{n}$ diode electrical behavior in a circuit simulator environment.

\section{REFERENCES}

[1] R. H. Caverly and M. Quinn, "A SPICE model for simulating the impedance-frequency characteristics of high frequency PIN switching diodes," in Proc. IEEE Int. Symp. Circuits Syst., 1999, vol. 6, pp. 282-285.

[2] D. Leenov, "The silicon PIN diode as a microwave radar protector at megawatt levels," IEEE Trans. Electron Devices, vol. ED-11, no. 2, pp. 53-61, Feb. 1964.

[3] R. H. Caverly and G. Hiller, "The small signal ac impedance of gallium arsenide and silicon $\mathrm{p}-\mathrm{i}-\mathrm{n}$ diodes," Solid State Electron., vol. 33, no. 10, pp. 1255-1263, 1990.

[4] R. C. Varshney, D. J. Roulston, and S. G. Chamberlain, "Some properties concerning the ac impedance of p-i-n and p-n- $n^{+}$diodes," Solid State Electron., vol. 17, pp. 699-706, 1974.

[5] F. Berz, R. W. Cooper, and S. Fagg, "Recombination in the end regions of pin diodes," Solid State Electron., vol. 22, pp. 293-301, 1979.

[6] R. H. Caverly and X. Ma, "Nonlinear stored charge vs dc bias-current relationship under high-level injection in pin diodes," Solid State Electron., vol. 32, no. 4, pp. 329-332, 1989.

[7] A.G.M. Strollo and P. Spirito, "A new PiN diode modelling approach for power electronic PSPICE simulations," in Proc. 25th Annu. IEEE Power Electron. Spec. Conf., Jun. 1994, vol. 1, pp. 52-58.

[8] W. Reiss, "Volterra series representation of a forward-biased p-i-n diode," IEEE Trans. Electron Devices, vol. 28, no. ED-12, pp. $1495-1500$, Dec. 1981

[9] R. H. Caverly, "RF technique for determining the ambipolar carrier lifetime in pin RF switching diodes," Electron. Lett., vol. 34, no. 23, pp. 2277-2278, Dec. 1998 